\title{
CHD1L prevents lipopolysaccharide-induced hepatocellular carcinomar cell death by activating hnRNP A2/B1-nmMYLK axis
}

Guangliang Wang ${ }^{1,2,4}$, Xiaofeng Zhang ${ }^{1,4}$, Wei Cheng ${ }^{3}$, Yanxuan Mo ${ }^{3}$, Juan Chen ${ }^{3}$, Zhiming Cao ${ }^{1}$, Xiaogang Chen ${ }^{1,3}$, Huiqin Cui ${ }^{1,3}$, Shanshan Liu ${ }^{3}$, Li Huang ${ }^{3}$, Ming Liu (iD) ${ }^{1,3}$, Lei Ma ${ }^{1}$ and Ning-Fang Ma (iD ${ }^{1,3 凶}$

(c) The Author(s) 2021, corrected publication 2022

Chromodomain helicase/ATPase DNA-binding protein 1-like gene (CHD1L) has been characterized to be a driver gene in hepatocellular carcinoma (HCC). However, the intrinsic connections between CHD1L and intestinal dysbacteriosis-related inflammation reaction in HCC progression remain incompletely understood. In this study, a specific correlation between CHD1L and nonmuscle isoform of myosin light chain kinase (nmMLCK/nmMYLK), a newly identified molecule associated NF-KB signaling transduction, was disclosed in HCC. CHD1L promotes nmMYLK expression and prevents lipopolysaccharide (LPS) induced tumor cell death. In vitro experiment demonstrated that overexpressed nmMYLK is essential for CHD1L to maintain HCC cell alive, while knocking down nmMYLK significantly attenuate the oncogenic roles of CHD1L. Mechanism analysis revealed that nmMYLK can prevent Caspase-8 from combining with MyD88, an important linker of TLRs signaling pathway, while, knocking down nmMYLK facilitate the MyD88 combines with Caspase- 8 and lead to the proteolytic cascade of Caspase as well as the consequent cell apoptosis. Mechanism analysis showed that CHD1L promotes the nmMYLK expression potentially through upregulating the heterogeneous nuclear ribonucleoproteins A2/B1 (hnRNP A2/B1) expression, which can bind to myosin light chain kinase $(M Y L K)$ pre-mRNA and lead to the regnant translation of nmMYLK. In summary, this work characterizes a previously unknown role of CHD1L in preventing LPS-induced tumor cell death through activating hnRNP A2/B1-nmMYLK axis. Further inhibition of CHD1L and its downstream signaling could be a novel promising strategy in HCC treatment.

Cell Death and Disease (2021)12:891; https://doi.org/10.1038/s41419-021-04167-9

\section{INTRODUCTION}

Dysbacteriosis, characterized by excessive lipopolysaccharideinduced endotoxemia and activation of TLRs-NF-KB signaling, might occur after antibiotic therapy, surgery, chemotherapy, or radiotherapy of cancer. Increasing evidence suggested that dysbacteriosis was associated with the progression of tumor. Chromodomain helicase/adenosine triphosphatase DNA-binding protein 1-like gene $(C H D 1 L)$ is originally verified in human hepatocellular carcinoma cells (HCC) and its oncogenic roles have been characterized in previous studies [1, 2]. Many studies revealed the oncogenic roles of CHD1L in different kinds of malignancies, such as ovarian carcinoma [3], bladder cancer [4], colorectal carcinoma [5], breast cancer [6], and lung adenocarcinoma [7], etc. Considering that dysbacteriosis is common in cancer patients, it is important to know how CHD1L works in the pathological microenvironment of dysregulated flora.

Myosin light chain kinase (MLCK/MYLK) has been complicated in tumor cell proliferation, invasion, and distant metastasis [8-10]. MYLK gene encodes mainly two different catalytic protein isoforms. The short isoform named smooth muscle MYLK (smMYLK) is mainly expressed in smooth muscle tissue, while the long isoform generally exists in nonmuscle tissues (nmMYLK) [11]. Recent study shows that nmMYLK involves in NF-KB- dependent transcription of inflammation related genes in endothelial cells [12]. Our unpublished high-throughput sequencing data indicated that nmMYLK was closely correlated with CHD1L expression in HCC, which hints a potential relationship between CHD1L and NF-KB signaling transduction.

In this study, the correlations between CHD1L and nmMYLK and their roles to tumor cells under LPS mimetic inflammatory condition were investigated, and nmMYLK was found to play an important role in CHD1L-promoted tumor cell proliferation and antiapoptotic effects. CHD1L upregulates heterogeneous nuclear ribonucleoproteins A2/B1 (hnRNP A2/B1) expression, which binding to MYLK pre-mRNA and leads to the regnant translation of nmMYLK. These findings suggested that CHD1L-regulated hnRNP A2/B1/nmMYLK axis was important in preventing LPSinduced tumor cell death. Blocking this critical axis might be a promising strategy in HCC treatment.

\section{RESULTS}

CHD1L promotes nmMYLK expression in HCC

To further study the mechanism of CHD1L, we have constructed the CHD1L overexpressed HCC cell line. QGY-7703 cells were transfected with CHD1L-GFP or Vector plasmid, the total RNA of

\footnotetext{
${ }^{1}$ Affiliated Cancer Hospital and Institute, Guangzhou Medical University, Guangzhou, China. ${ }^{2}$ Department of Histology and Embryology, Faculty of Basic Medical Sciences, Guilin Medical University, Guilin, Guangxi, China. ${ }^{3}$ Guangzhou Municipal and Guangdong Provincial Key Laboratory of Protein Modification and Degradation, School of Basic Medical Sciences, Guangzhou Medical University, Guangzhou, China. ${ }^{4}$ These authors contributed equally: Guangliang Wang, Xiaofeng Zhang. ${ }^{凶}$ email: nfma@gzhmu.edu.cn Edited by Professor Anastasis Stephanou
} 
CHD1L-GFP or Vector stable cell lines were extracted and checked for high-throughput sequencing. By analyzing the throughput mRNA sequencing database, we identified MYLK as a potential downstream gene of CHD1L (unpublished data). To confirm the relationship between CHD1L and MYLK, the endogenous expression of CHD1L and MYLK in different HCC cell lines including LO2, QGY-7703, HepG2, Huh7 was measured by qRT-PCR and western blot (Supplementary Fig. S1). Similar trends in CHD1L and MYLK expression were detected from qRT-PCR analysis (Supplementary information, Fig. S1A). Western blot analysis indicated smMYLK expression levels in all tested cell lines were similar; however, an additional nmMYLK band was appeared in HepG2, Huh7 cells, the cells possess a high level expression of CHD1L (Supplementary information, Fig. S1B), indicated the nmMYLK, rather than smMYLK, was tightly correlated with CHD1L expression. As a result, we further examined the correlation between CHD1L and nmMYLK in 73 paired HCC specimens. Overexpression of CHD1L and nmMYLK was observed in 64/73 (87\%) and 43/73 (59\%) HCC samples, when compared with the para-nontumorous counterparts (Fig. 1A, B). A positive correlation was shown using Spearman's correlation analysis ( $87 \%$ vs. $59 \%$, Spearman correlation coefficient, 0.5142; $P<0.0001$ ) (Fig. 1C). The correlation between MYLK expression and clinicopathological features of 73 HCC patients was evaluated. High MYLK expression was positively correlated with HBsAg $(P=0.04)$, microvascular invasion $(P=$ $0.031)$, and tumor encapsulation $(P=0.028)$ (Table 1$)$. Western blot results from some of the paired HCC specimens showed that the expression of nmMYLK was particularly prominent in the tumor specimens with CHD1L overexpression, as compared with the basal level of smMYLK (Fig. 1D). To further confirm this relationship, CHD1L-specific small-interfering RNA (siRNA) was used to imitate CHD1L downregulation in HepG2. And the expression of nmMYLK was substantially reduced corresponding to the downregulation of $\mathrm{CHD} 1 \mathrm{~L}$ as compared with the nonspecific siRNA control (Fig. 1E, F).

An alternative approach was applied by transfecting 7703 cells with CHD1L expression plasmid. Upregulation of CHD1L increased the expression of MYLK in 7703 cells. Similar to that Supplementary Fig. S1B, MYLK isoforms were expressed differently in responding to the $\mathrm{CHD} 1 \mathrm{~L}$ upregulation. Besides the basal expression of smMYLK, nmMYLK was specifically expressed in 7703-CHD1L cells, as compared with its vector control (Fig. 1G, H). These results suggest a positive regulatory role of $\mathrm{CHD} 1 \mathrm{~L}$ on nmMYLK expression.

\section{The essential role of $\mathrm{nmMYLK}$ in promoting the proliferation and antiapoptosis of HCC cells}

Considering the special anatomical environment of the liver and the involvements of nmMYLK in NF-KB-dependent transcription of inflammatory or survival related genes, we applied exogenous LPS to the culture system to mimic the inflammatory environment. To assess the general effect of nmMYLK on malignant phenotype of HCC cells and to understand its mechanism, we transduced HepG2 with shRNAs targeting $n m M Y L K$ (hereafter referred to as shnmMYLK) or a non-targeting shRNA (hereafter referred to as shCTR). An alternative approach was applied by transfecting 7703 cells with $n m M Y L K$ expression plasmid. Accordingly, the expression of $n m M Y L K$ was determined by using qRT-PCR and Western blotting. And down or upregulation of nmMYLK in HepG2 or 7703 cells was significantly more efficient than their vector controls (Fig. 2A, B). MTS analysis was applied to evaluate the effects of nmMYLK on HCC cells proliferation. The results indicated that downregulation of nmMYLK inhibited HepG2 cell proliferation with or without LPS treatment (Fig. 2C, Left). On the contrary, the enforced expression of nmMYLK increased tumor cells proliferation with or without LPS treatment (Fig. 2C, Right). Similar results were observed in the colony formation assay with that of MTS assay (Fig. 2D).
Downregulation of nmMYLK induced potent suppression on HepG2 cells migration. After treated with LPS, the migration cells/ field in both the shCTR and nmMYLK downregulated cells (shnmMYLK) were increased, but the latter was lower than that of the former (Fig. 2E). Overexpressed nmMYLK in 7703 cells significantly reversed this phenotype when compared with the vector control cells, and this effect was enhanced after LPS treatment (Fig. 2F).

To explore the molecular mechanism involved in nmMYLK mediated tumor cell survival, the effects of nmMYLK on apoptosis were further evaluated. Flow Cytometry assays revealed that downregulated nmMYLK could enhance apoptosis. The apoptotic indexes were $0.1 \%$ and $3.4 \%$ or $1.6 \%$ in HepG2-shCTR cells and HepG2-shnmMYLK cells, respectively. LPS reduced the apoptosis of tumor cells, but the apoptosis rate reminded higher in HepG2shnmMYLK cells than that of control (Fig. 3A, Left). No significant difference was observed in the apoptotic index of 7703-nmMYLK and 7703-vector cells with and without LPS treatment (Fig. 3A, Right).

The expression levels of cleaved Caspase-9, Caspase-8, Caspase3 , and Cyclin D1 in aforesaid cell lines was detected and the results showed that the cleaved Caspase-3/8 was increased in HepG2shnmMYLK cells and Cyclin D1 experienced a greater reduction than that of control cells when treated with or without LPS (Fig. 3B, Left and Supplementary Fig. S2A). No significant apoptosis was found in 7703 Vector or nmMYLK cells, which mean the lower basal apoptosis level, and weak cleaved Caspase-3/8 bends were only detected after Chx + TNF-a treatment. And the expression of Cyclin D1 was clearly upregulated in nmMYLK overexpressed 7703 cells as compared with its vector control (Fig. 3B, Right and Supplementary Fig. S2B).

The tumorigenic effect of nmMYLK was verified in nude mice through subcutaneous implantation of HepG2-shnmMYLK and its control HepG2-shCTR cells. The size and weight of the tumor from the shnmMYLK-transfected HepG2 cells were smaller than that of control $\left(990.56 \pm 401.38 \mathrm{~mm}^{3}\right.$ vs $61.67 \pm 94.69 \mathrm{~mm}^{3}, \quad P<0.01$; $0.69 \pm 0.25 \mathrm{~g}$ vs $0.06 \pm 0.08 \mathrm{~g}, P<0.01$ ) (Fig. 3C, D). The expression of Cyclin D1 reduced and cleaved Caspase-3/8 increased obviously in HepG2-shnmMYLK group, comparing with the control group (Fig. 3E). To summarize, the results from gain- or loss-of-function studies indicate that nmMYLK mediated tumor cell proliferation and apoptosis.

\section{nmMYLK is essential for CHD1L-promoted malignant proliferation in tumor cells}

To clarify the importance of nmMYLK for CHD1L to promote the malignant proliferation of HCC by infecting 7703-CHD1L cells with shnmMYLK lentivirus (refered as 7703-CHD1L-shnmMYLK), the expressions of nmMYLK and CHD1L were evaluated (Fig. 4A). MTS assay showed that LPS promoted the proliferation of 7703-CHD1L cells and its vector control, and downregulation of nmMYLK inhibited CHD1L-induced cell proliferation. LPS could not rescue the effects of the downregulation of nmMYLK on cell proliferation (Fig. 4B). The clone formation assay got the same conclusion (Fig. 4C). Flow Cytometry assay revealed a lower apoptotic index from CHD1L overexpressed cells than that of vector control $(0.3 \%$ vs $1.7 \%)$. while, knockdown nmMYLK increased the apoptosis indexes in $7703-$ CHD1L cells (0.5\% up to $1.3 \%$ ) (Fig. 4D). Transwell assays showed that downregulation of nmMYLK in 7703-CHD1L cells significantly decreased cell migration, which could not be rescued by an additional treatment of LPS when compared with 7703-CHD1L cells (Fig. 4E). To confirm the in vivo effects of nmMYLK on CHD1L-promoted tumor growth, 7703-Vector, 7703CHD1L, and 7703-CHD1L-shnmMYLK cell line were constructed and applied for subcutaneous implantation in nude mice. As shown in Fig. 4F, G, knockdown nmMYLK attenuated the tumorigenic capacity of CHD1L. Tunnel and western blot analysis showed increased apoptosis rates in 7703-CHD1L-shnmMYLK 
A

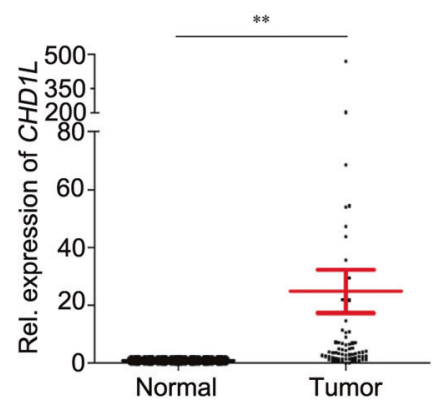

D

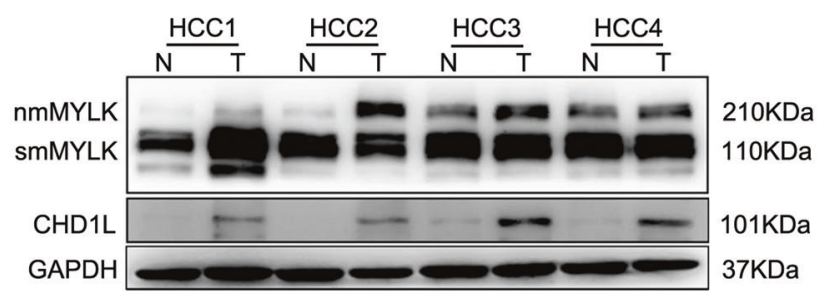

F

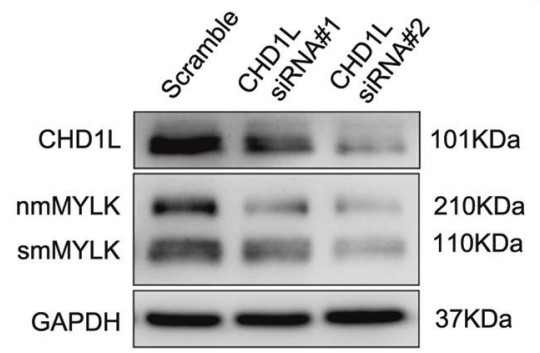

B

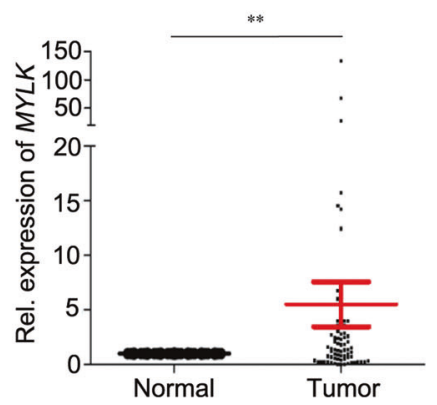

$\mathrm{E}$
C
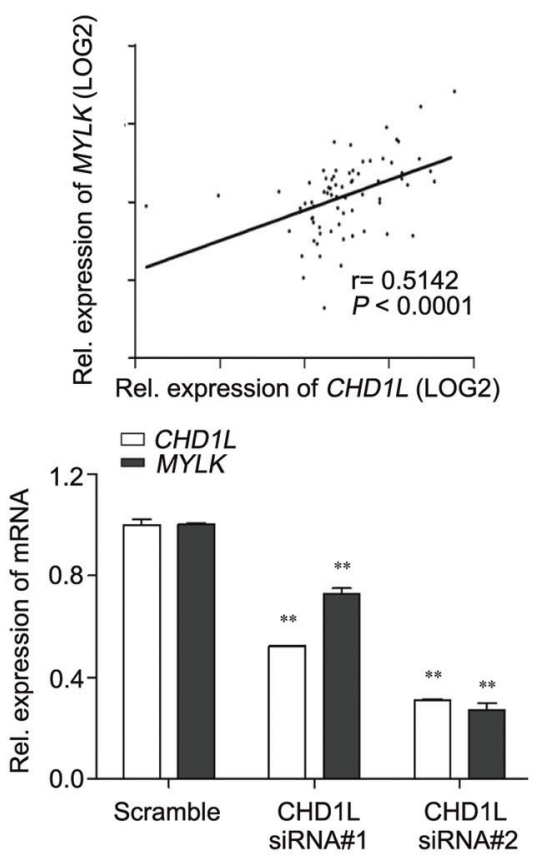

$\mathrm{H}$
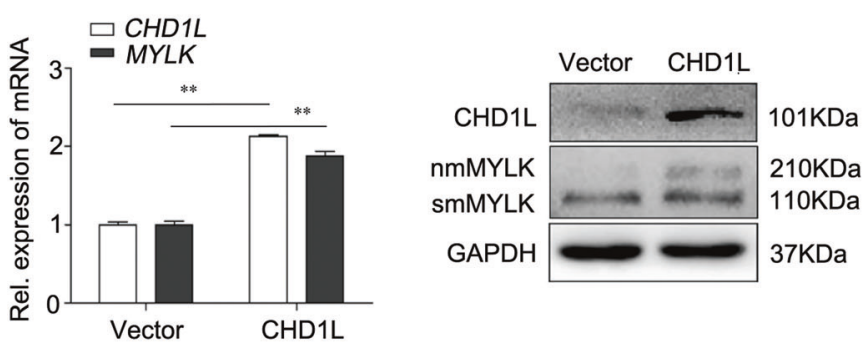

Fig. 1 CHD1L promotes nmMYLK expression in HCC. A, B Quantitative RT-PCR (qRT-PCR) analysis for CHD1L and MYLK in 73 pairs of HCC tumors (T) and adjacent nontumor tissues $(\mathrm{N})$. Data shown as mean \pm SD. C Spearman correlation analysis for the expression of CHD1L and MYLK $(r=0.5142, P<0.0001)$, based on qRT-PCR results. D Western blot assay was used to detect the expression of CHD1L and MYLK in paired HCC samples, with GAPDH as normalized control. E, F The mRNA and protein levels of CHD1L and MYLK in HepG2 cells were detected with qRT-PCR and western blot after siCHD1L treatment, Data were performed by mean with SD from three independent experiments. G, $\mathbf{H}$ The mRNA and protein levels of CHD1L and MYLK in 7703 cells were detected using qRT-PCR and western blot. Data were performed by mean with SD from three independent experiments. ${ }^{* *} P<0.01$.

implantation group (Fig. 4H and Supplementary Fig. S3). These results indicated that nmMYLK is essential for CHD1L-promoted malignant growth.

\section{CHD1L-mediated nmMYLK expression was depended on hnRNP A2/B1}

HnRNPs are a class of multifunctional proteins belonging to the heterogeneous ribonuclear protein family, and involve in processing the heterogeneous nuclear RNA into messenger RNA [13, 14], we've accidentally found that the heterogeneous nuclear ribonucleoprotein A2/B1 (hnRNP A2/B1) was upregulated in CHD1L overexpression cells (Fig. 5A). A core component being hnRNP A2/ B1 that is involved in alternative splicing $[15,16]$, and it was reported that hnRNP A1 is involved in the alternate splicing of MYLK [17]. The effect of hnRNP A2/B1 on MYLK splicing was validated by transfecting HepG2 cells with hnRNP A2/B1-specific siRNA, Western blot indicated that knockdown hnRNP A2/B1 could inhibit the expression of nmMYLK, while the expression of smMYLK was upregulated (Fig. 5B, C). Previous studies showed that hnRNP A2/B1 specifically binds to UAGGG sequences $[18,19]$.
We analyzed the full-length DNA and protein sequences of MYLK, and found there is a TAGGG motif in the exon 3-4 and ATG in the exon 15 of MYLK with $\sim 2800$ bps length. MYLK protein domain analysis showed that nmMYLK possess a specialized N-terminal domain containing 922-aa, which cover the motif ranged from exon 1 to exon 15, contains more $2766 \mathrm{bps}$ of nucleic acid sequence than that of smMYLK (Fig. 5D). RNA Immunoprecipitation (RIP) experiment further verified that hnRNP A2/B1 indeed binds to the pre-mRNA of $M Y L K$, more exactly, its binding site was at the exon 3-4 region (Fig. $5 \mathrm{E}$ ). The above results indicated that CHD1L promotes nmMYLK formation via upregulating hnRNP A2/ $B 1$, which can bind to the pre-mRNA of MYLK and protect it from splicing.

\section{nmMYLK is essential for LPS-induced TLR4/NF-KB signal transduction}

nmMYLK was involved in NF-KB-dependent inflammatory signal transduction and promoted the transcription of surviving genes in vascular endothelial cells $[20,21]$. We next investigated whether the same effects of nmMYLK were existed in tumor cells. Previous 
Table 1. Association of MYLK expression with clinicopathological characteristics in 73 HCC samples.

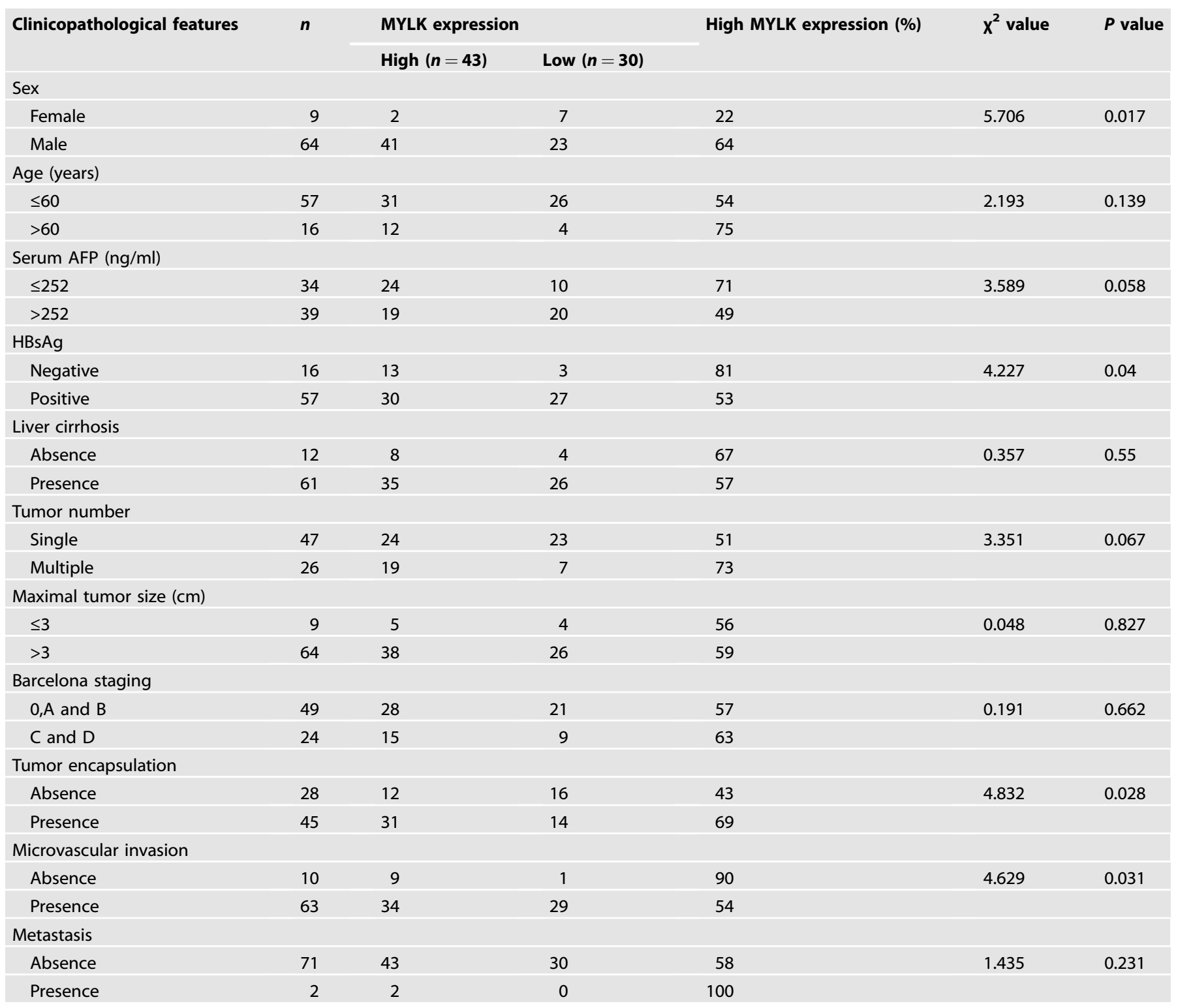

study has shown that the LPS induces $\mathrm{Ca}^{2+}$ entry in a TLR4dependent manner and the internal flow of $\mathrm{Ca}^{2+}$ could activate nmMYLK [12]. In this regard, we applied Fura-4-AM to determine cytosolic $\mathrm{Ca}^{2+}$ concentration in responding to LPS. And found that LPS caused an increased intracellular $\mathrm{Ca}^{2+}$ concentration in HepG2 cells (Supplementary Fig. S4A). To validate LPS induces nmMYLK enzymatic activity, we observed that phosphorylated MLC2 was detected after LPS treatment, which representing the activation of nmMYLK (Supplementary Fig. S4B). Considering the aforementioned effects of LPS on nmMYLK in tumor cells, we speculated that, as the receptor of LPS, TLR4, and its downstream NF-KB signaling pathway should be involved. Then, the activity of the key subunit of NF-KB, the phosphorylated p65 and its downstream target genes (DTGs) expression in HepG2-shnmMYLK cells were detected. Western blot and immunofluorescence assay indicated that LPS induced p65 phosphorylation and nuclear translocation (Fig. 6A, B). No significant differences in the nmMYLK downregulated cells were found (Fig. 6A, B). LPS increased the transcription of NF-KB DTGs, such as Cyclin D1, CIAP1, ICAM-1, NFATC1, EHD1, and SDC4 in HepG2 cells had been reported previously [22]. Herein, as compared to HepG2-shCTR cells, only a slight increase was observed in HepG2-shnmMYLK cells (Fig. 6C), indicating that nmMYLK is essential for LPS-induced NF-KB activation.

To ascertain the possible link between nmMYLK related NF-kB activation and MyD88-IRAK4 complex, lysates from LPS treated HepG2 cells were immunoprecipitated with anti-nmMYLK antibody, followed by immunoblotting with anti-MyD88 and antiIRAK4 antibody to evaluate their interactions. The results found that nmMYLK could combine with MyD88 and IRAK4, which was strengthened after LPS treatment (Fig. 6D). Likewise, using antiMyD88 antibody, unexpected results showed that besides nmMYLK and IRAK4, the apoptotic protein Caspase-8 also interacted with MyD88 and oppositely correlated with nmMYLK abundance, and knockdown nmMYLK enabled MyD88 to interact with Caspase-8 (Fig. 6E). To confirm whether MyD88 interacts with Caspase-8, anti-Caspase-8 antibody was used to repeat coimmunoprecipitation. Similar results were obtained, which indicated a real binding between Caspase- 8 and MyD88. However, no results showed a possible binding between Caspase- 8 and 
A

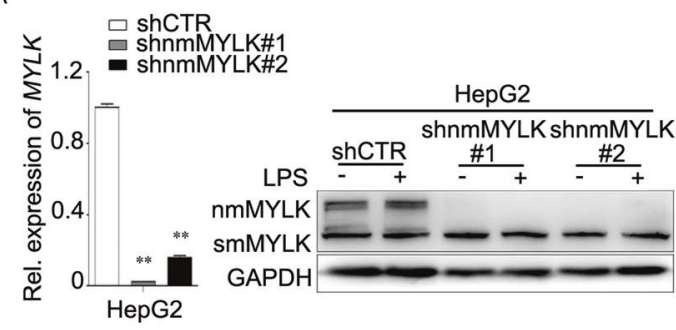

C
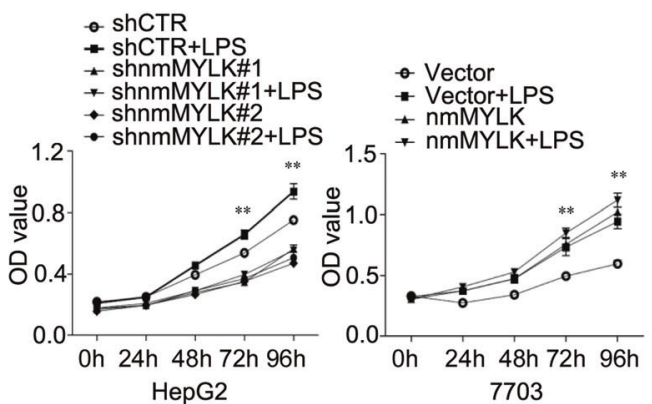

E

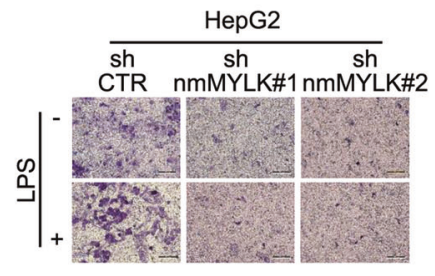

B

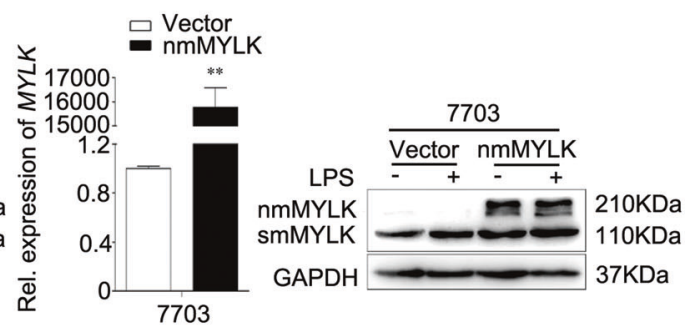

D

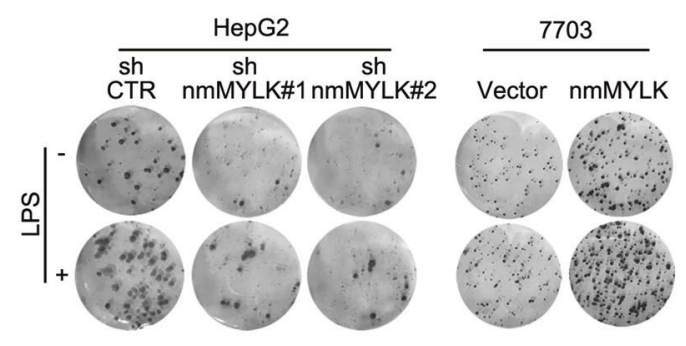

F
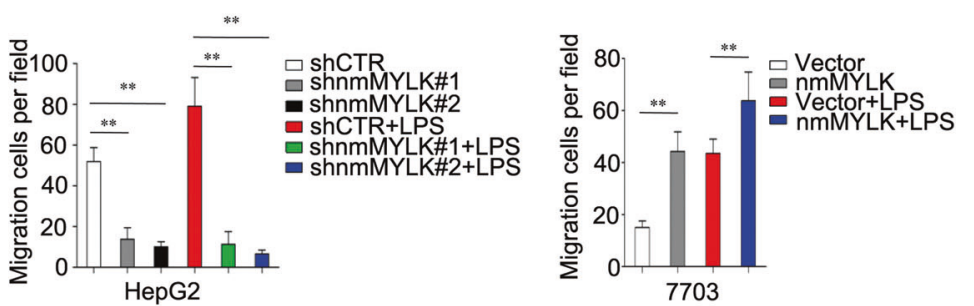

Fig. 2 nmMYLK promote the proliferation of HCC cells. A-F HepG2 cells were transfected with shRNA-nmMYLK lentivirus or lentivirus-vector control before LPS treatment, 7703 cells were transfected with nmMYLK expression constructs or its vector before LPS treatment. The expressions of MYLK in HepG2 and 7703 cells were detected using qRT-PCR and western blot (A, B). Cell proliferation abilities were examined using MTS assay (C) and Colony formation assay (D). Cell migration abilities were examined using Transwell test (E, F). The experiments were repeated three times independently, and the bars represent SD. ${ }^{* *} P<0.01$.

nmMYLK or IRAK4. Downregulation of nmMYLK increased the interaction of Caspase-8 with MyD88 (Fig. 6F). The above results indicated a new mechanism of CHD1L in preventing LPS-induced tumor cell death via activating hnRNP A2/B1-nmMYLK axis (Fig. 7).

\section{DISCUSSION}

As one of SNF2 superfamily member, CHD1L possessing putative helicase sequence motifs is similar to those found in helicase superfamily 2, which confers the ability for stabilizing or perturbing protein-DNA interaction [23]. CHD1L protein contains two helicase domains, a C-terminal macro domain and a nuclear localization sequence, which function as a transcription regulator to affect a broad spectrum of cellular processes [24]. CHD1L regulates downstream genes transcription by directly binding to the promoters of related target gene, such as ARHGEF9, TCTP, Nur77, and SPOCK1 to maintain HCC cell survival, migration, invasion, and inhibits apoptosis [25-28]. Here, we demonstrate that the nmMYLK expression is closely depending on CHD1L in HCC. Overexpression of nmMYLK was detected in over $59 \%$ of HCC cases with a positive correlation with CHD1L expression.

MYLK and its spliced isoforms play important roles in regulating cell survival, proliferation, and motility $[29,30]$. MYLK has been reported to phosphorylate myosin light chains (MLC) and regulate the invasion and metastasis of HCC and some other malignancies, which were mainly focused on MYLK subtype SmMYLK (108-130 kD) [31]. In this study, the results from in vitro and in vivo validation proved that nmMYLK, not smMYLK, is essential in CHD1L driven HCC cancer malignant phenotype, noticeable, all of these effects partially depended on LPS mimicked dysbacteriosis situation.

Previous studies demonstrated that nmMYLK was relates to NF$\mathrm{KB}$-dependent inflammatory and the transcription of survival genes in acute lung injury [21,32]. Herein, we confirmed that nmMYLK promotes HCC cell proliferation by activating NF-KB signaling and upregulating its downstream genes Cyclin D1, CIAP1, ICAM-1, NFATC1, EHD1, and SDC4 that are correlated with the malignant phenotype of some kinds of tumors. This indicated that nmMYLK plays a specific role in LPS-induced malignancies.

Apoptosis inhibition is associated with tumor development. Caspase-8, executor of apoptosis, influences cell adhesion and migration through post-cleavage remodeling of cytoskeleton and signaling elements that control focal adhesion turnover [33]. Our study showed that knocking down nmMYLK increased the accumulation of cytoplasmic Caspase- 8 and trigger the proteolytic cascade reaction. However, the same effects were not observed in nmMYLK overexpressed 7703 cells. It might be due to the suppression of apoptosis-related genes in tumor cells. Accumulation of cleaved Caspases- 8 and Caspases- 3 induced by downregulated nmMYLK led to cell death, in addition, the oncogenic effects of CHD1L on HCC cells were significantly blocked, suggesting nmMYLK was critical for CHD1L. Both the gain- or loss-of function tests supported that the CHD1L associated oncogenic role of nmMYLK was closely correlated with Caspase-8 inactivation. 
A

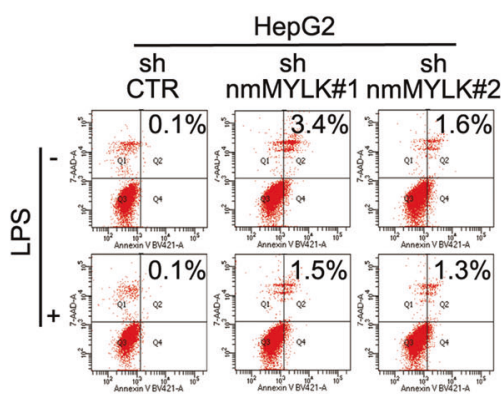

B

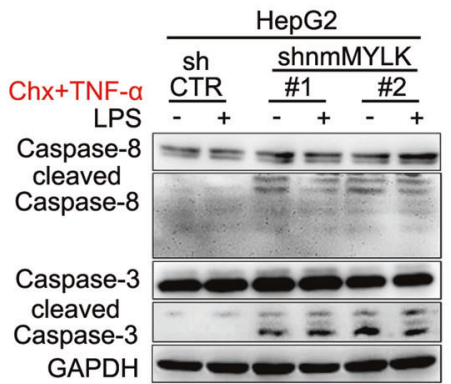

C

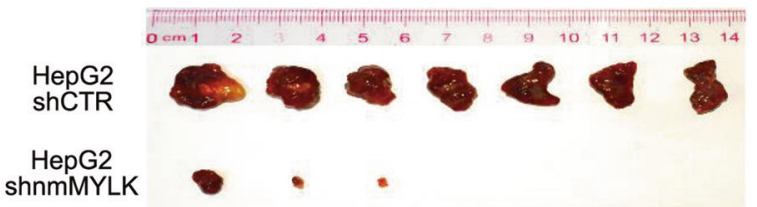

D
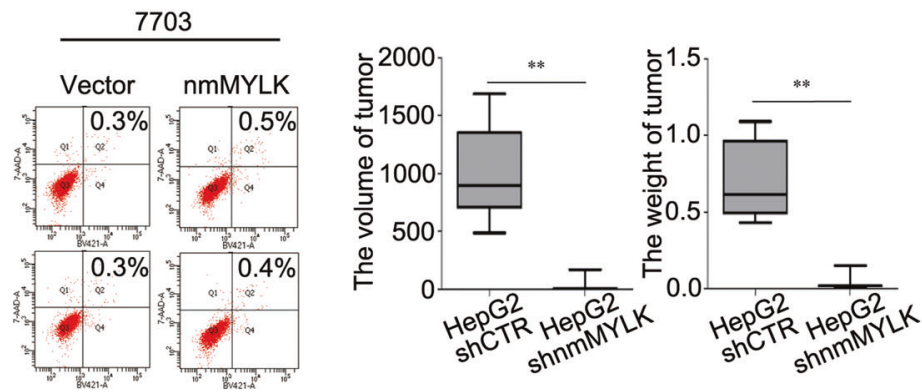

E
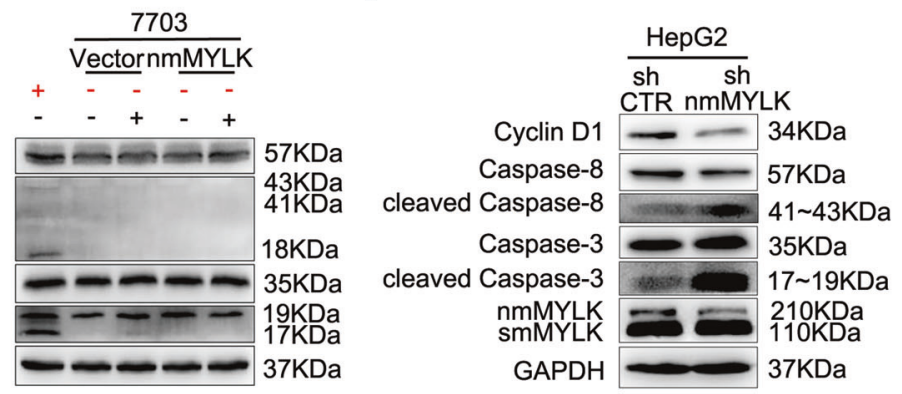

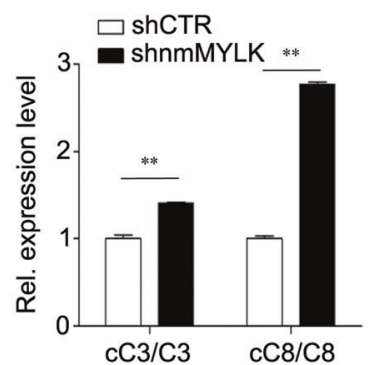

Fig. 3 Downregulation of nmMYLK in HCC cells promotes tumor cells apoptosis and attenuates tumor formation in nude mice. A, B HepG2 cells were transfected with shRNA-nmMYLK lentivirus or lentivirus-vector control before LPS treatment, 7703 cells were transfected with nmMYLK expression constructs or its vector before LPS treatment. Flow cytometry $(\mathbf{A})$ and western blot (B) were used for examining the apoptosis rate of HepG2 and 7703 cells. C, D Mice xenograft models were constructed by inoculated HepG2 cells subcutaneously. Tumors dissected from different groups (C), tumor volumes and weight analysis (D). E Relative cascade reactions of caspases in xenograft tumors were examined using western blot. Data were performed by mean with SD from three independent experiments. ${ }^{* *} P<0.01$.

Heterogeneous nuclear ribonucleoproteins (hnRNPs) plays an essential role in regulating gene expression, including RNA splicing, polyadenylation, etc $[34,35]$. hnRNP A2/B1 was implicated in the progress of breast cancer, head and neck cancer, non-small cell lung cancer, and prostate cancer [15, 36-38]. Recent studies indicated hnRNP A2/B1 could interact with miR-503HG to control the metastasis of HCC and NF-KB signaling pathway [39]. Here, a positive correlation between CHD1L and hnRNP A2/B1 was identified. Specifically, CHD1L upregulated the expression of hnRNP A2/B1 in HCC cells. Meanwhile, knocking down hnRNP A2/B1 could reduce the expression ratio of nmMYLK/smMYLK. RIP experiments confirmed that hnRNP A2/B1 could bind to the motif of the MYLK pre-mRNA and lead to the long transcript of nmMYLK formation in HCC cells. Besides, there are two smMYLK and nmMYLK bands were observed in HepG2 and Huh7 cells in Fig. S1B. The possible reason is due to the high internal level of CHD1L, which upregulated hnRNP A2/B1 expression and subsequent control the alternate splicing of MYLK. Given these, we speculate that the two bands of smMYLK and nmMYLK probably belong to their different isoforms. Because hnRNP A2/B1 dominantly promotes the long isoform nmMYLK formation, logically, the cells that express high level CHD1L would generate more nmMYLK, and vice the versa. With the same reason, we can interpret that why the expression levels of smMYLK were lower in HepG2 and Huh7 cells than that in LO2 and 7703 cells, which is because the former expressed higher level of CHD1L than the latter one does.

In view of LPS-related reactions and the oncogenic roles of nmMYLK in HCC, the mechanism of nmMYLK for transmitting LPSTLR4 signaling to its downstream NF-KB pathway was undisclosed. It was evident that TLR4 delivered immune responses through activating its adaptor MyD88 and recruiting IRAK4, which then phosphorylates IRAK1 and IRAK2 to induce NF-KB activity [40]. Besides its role in mediating actomyosin, nmMYLK could also function as a scaffold mediating protein to promote protein interactions via its 922-aa N-terminal domain [41]. Consistent with the studies in endothelial cells [12], nmMYLK could interact with MyD88 and IRAK4 in HCC cells, this phenomenal was further strengthened by LPS treatment. Interestingly, MyD88 failed to bind IRAK4 in response to LPS in nmMYLK downregulated cells. These observations best fit a model that LPS may induce $\mathrm{Ca}^{2+}$ influx and activate nmMYLK consequently, which could function as a scaffold to support the interaction of MyD88-IRAK4, and finally promote the transcription of NF-KB downstream genes. It is known that the oligomerization of MyD88 death domain was critical for the interaction of MyD88 and IRAK4 [42]. Although nmMYLK has no canonical death domain, the serine/threonine residues in MyD88 domain might serve as the substrates of nmMYLK, which results in the oligomerization of MyD88 and its interaction with IRAK4 [42]. 
A

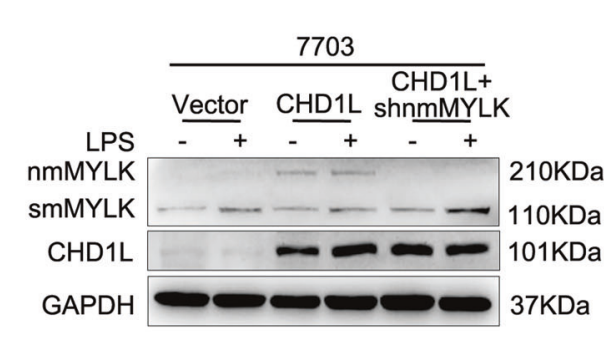

D

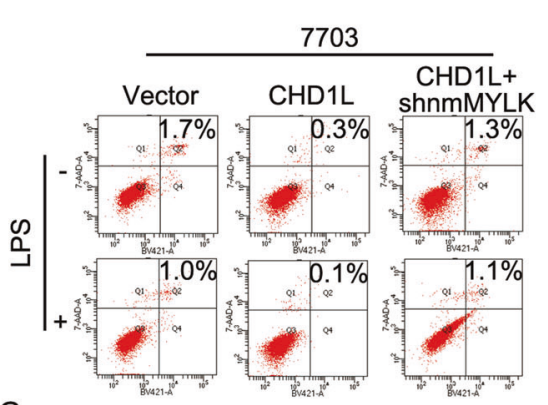

G

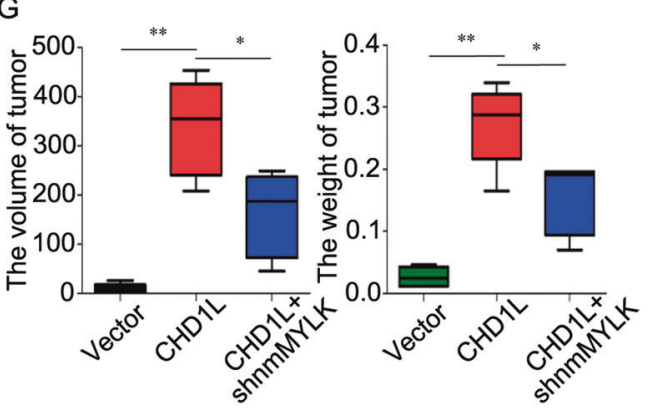

E
B

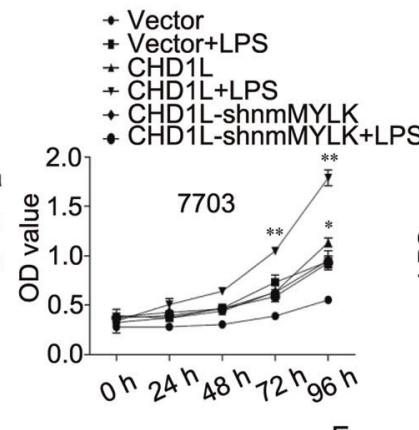

C

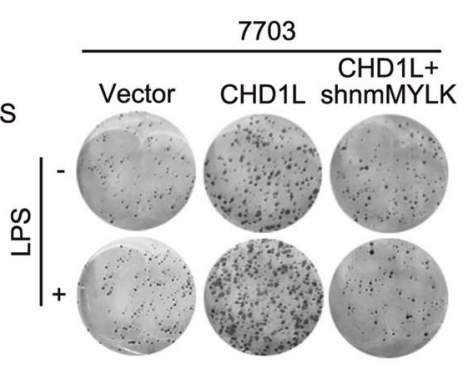

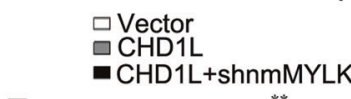
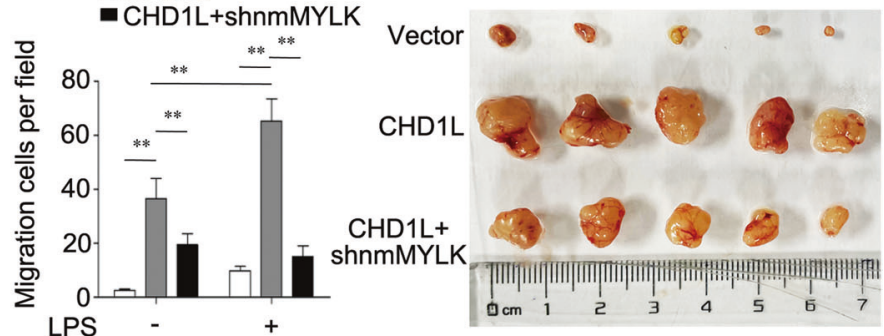

$\mathrm{H}$

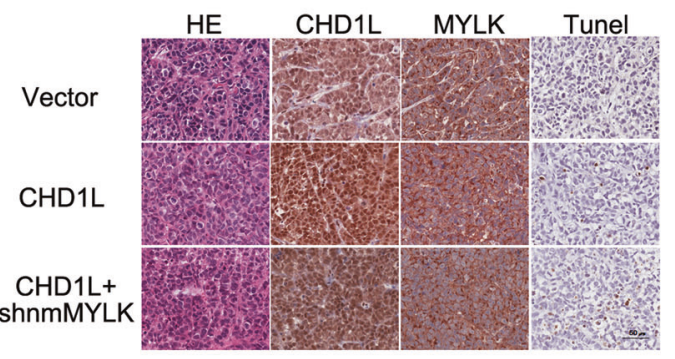

Fig. $4 \mathrm{nmMYLK}$ is essential for CHD1L-promoted malignant proliferation in tumor cells. A-H 7703 overexpression CHD1L cells were infected with specific shRNA (shnmMYLK) lentivirus. Western blot was applied to detect the expression of CHD1L and MYLK (A). Cell proliferation ability was examined using MTS assay (B) and Colony formation assay (C). Cell apoptosis level was examined using flow cytometry analysis (D). Cell migration ability was examined using Transwell test (E). 7703-CHD1L cells infected with shnmMYLK lentiviruses and inoculated subcutaneously into nude mice, following by monitoring for tumor growth, Tumor pictures (F), Tumor volumes and weight (G). Tumor tissues were embedded with paraffin and subjected to $\mathrm{H} \& \mathrm{E}$ and immunohistochemistry analysis $(20 \times)$, Scale bar $=50 \mu \mathrm{m}$, representative images were presented $(\mathbf{H})$. Data were performed by mean with SD from three independent experiments. ${ }^{*} P<0.05,{ }^{* *} P<0.01$.

Even so, the above reports were still insufficient to explain the effects of downregulated nmMYLK on cell apoptosis.

TLRs signal transduction through two main pathway: one is MyD88 combined with IRAK1/4 to activate NF-KB pathway, the other is MyD88 interacted with Fas-associated with death domain protein (FADD) and transmit signaling to Caspase-8 to induce apoptosis [43]. It can be deducted that downregulated nmMYLK might prevent MyD88 from binding to IRAK4, which gives the chance to MyD88 to integrate with FADD-Caspase-8. Lots of studies have proven that MyD88 could interacts with other proteins, although the mechanisms were not known completely [44]. Our study provides a possibility that the Caspase- 8 may bind to MyD88. Caspase- 8 contains two death effector domains (DED). When the ligands exist, the inhibitor of death domain on the receptor's intracellular peptide will dissociate and expose the intracellular peptide DD domains [45]. MyD88 also contains a DD domain and can offer a structural basis for interacting with Caspase-8, which has been recently proved to be involved in cell cytoskeletal remodeling and migration in a number of cell types [46]. Our study shows the role of nmMYLK in the interaction of MyD88 with IRAK4 or Caspase-8. We speculate that MyD88 could bind to Caspase- 8 and trigger the cascade of Caspase, which in turn activates downstream apoptotic signaling molecules, initiates cell apoptosis and inhibits cell migration. This mode clearly explains the oncogenic roles of nmMYLK in HCC cells.

Taken together, our results suggested that nmMYLK is a critical effector of CHD1L in response to LPS-induced TLR4/MyD88 signaling, which depends on the phenotype of HCC cells differently. Overexpression of nmMYLK worked synergistically with CHD1L to promote HCC cells proliferation. While, downregulation of nmMYLK blocked the malignant phenotype of HCC cells induced by LPS/ TLR4/MyD88/NF-KB signaling, promoted MyD88 binding with Caspase-8 to trigger cell apoptosis.

In summary, the results above suggest that nmMYLK functions as a key molecule for CHD1L to respond to LPS/TLR4/MyD88/NF$\mathrm{KB}$ signaling. And abolishment of CHD1L and nmMYLK might block the promotion of LPS on malignant cells proliferation, which might be a novel therapeutic strategy for HCC treatment.

\section{MATERIALS AND METHODS}

\section{Cell lines, tumor specimens}

LO2, QGY-7703, Huh7, and HepG2 cell lines were applied and their background information has been described in previous studies [28]. Cells were cultured in DMEM supplemented with $10 \%$ fetal bovine serum. LPS 
B

A
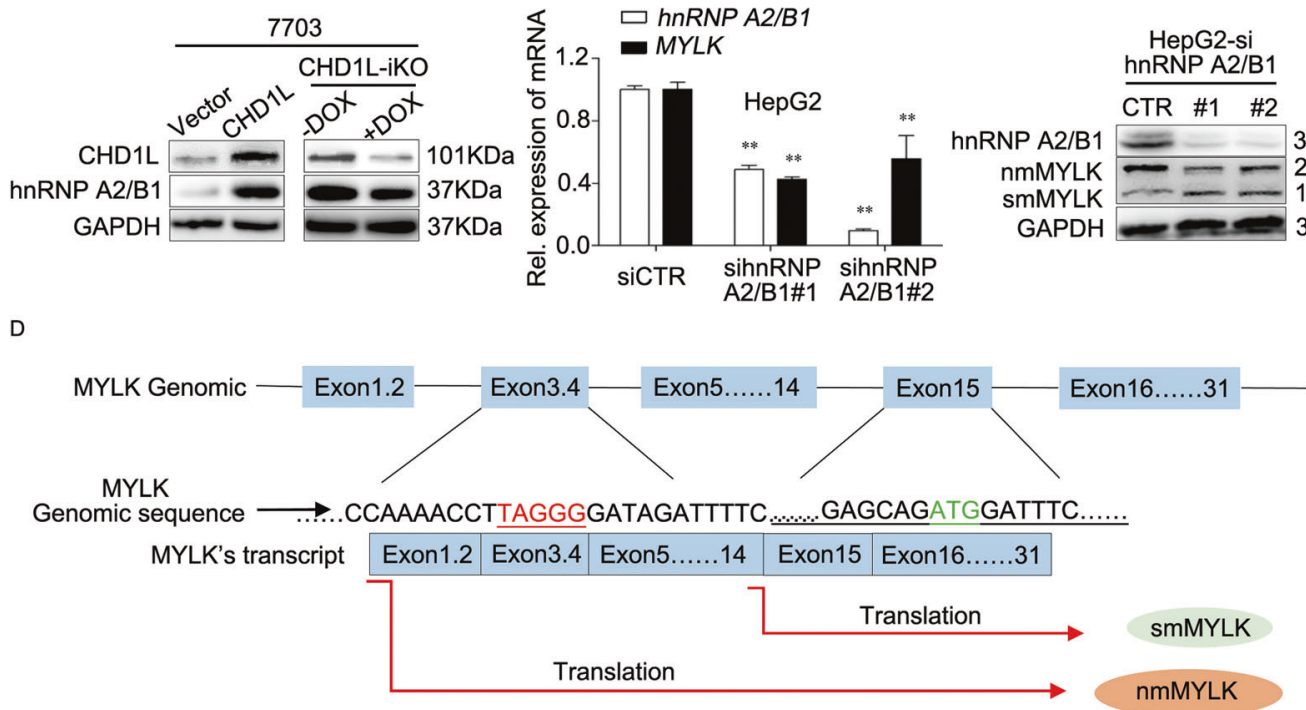

E

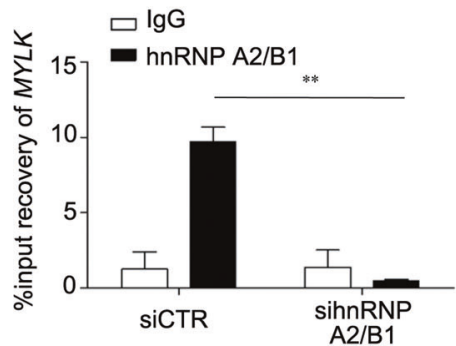

C

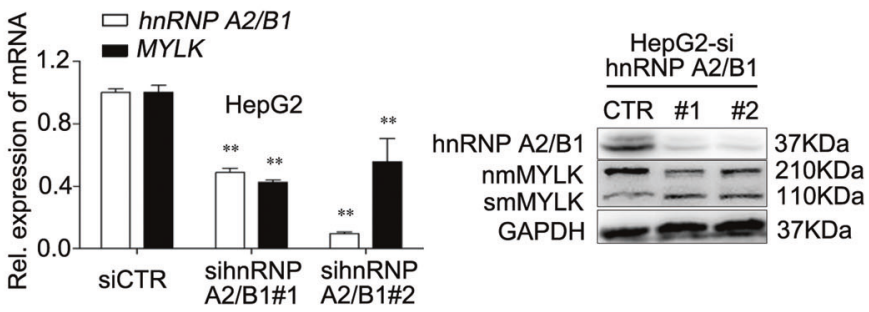

D

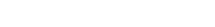


A

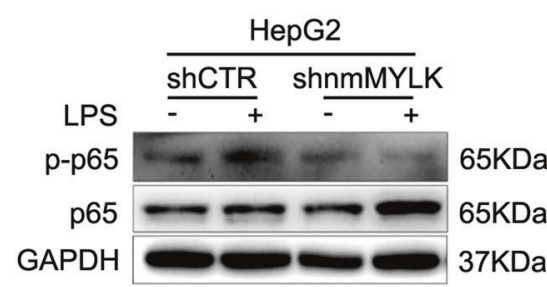

C

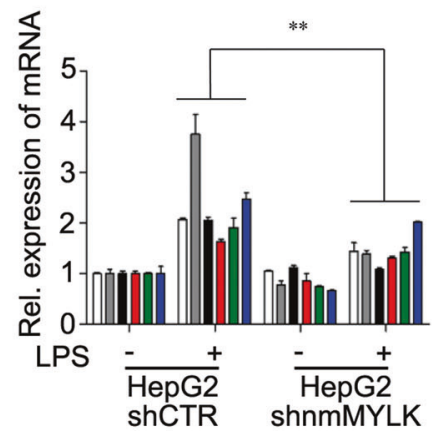

E

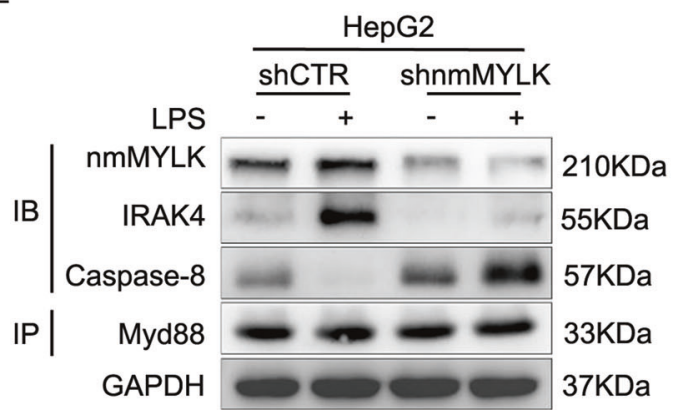

B

LPS

$\square$ CyclinD1

- clAP1

- ICAM-1

- NFATC1

- EHD1

- SDC4

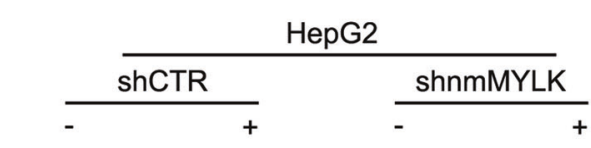

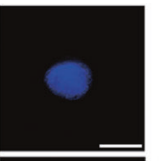
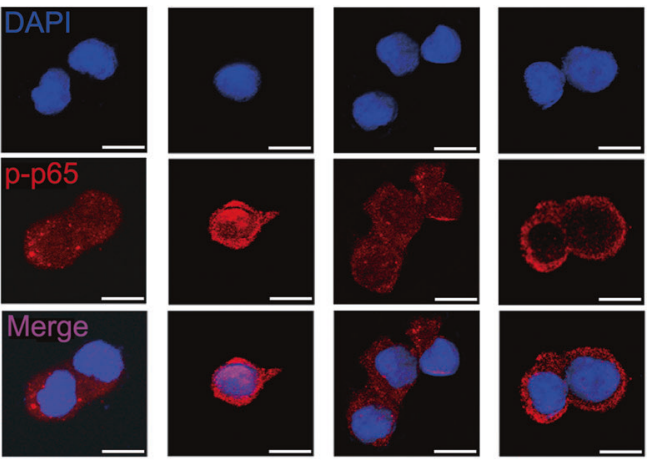

D
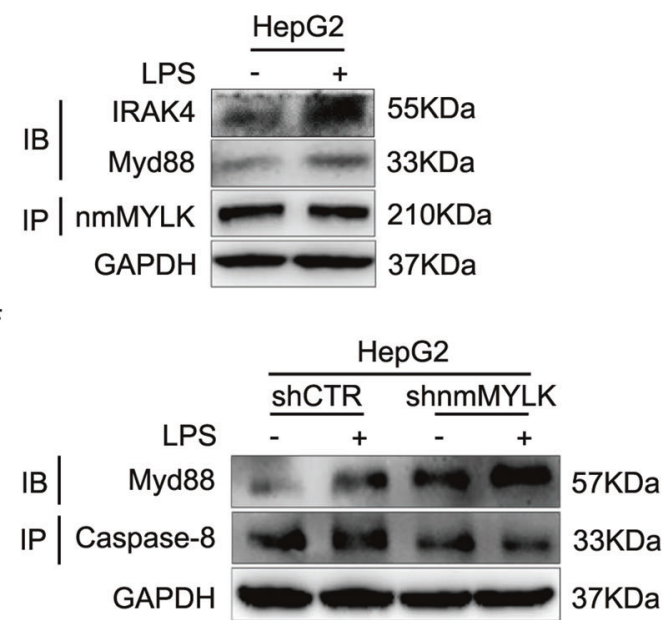

Fig. 6 nmMYLK is needed for LPS-induced NF-KB activation and apoptosis inhibition. A, B The expression and localization of p65 and p-p65 were detected by western blot and IF assays in HepG2 cells with or without LPS treatment. Scale bar $=10 \mu \mathrm{m}$. C The mRNA levels of Relative NF-KB DTGs were examined in HepG2 cells with or without LPS treatment. D The co-IP assay was carried out in HepG2 cells with or without LPS treatment for verifying the interaction between Myd88, IRAK4, and nmMYLK. E Related binding capacities of Myd88 to nmMYLK, IRAK4, and Caspase-8 were examined by co-IP assay in HepG2 cells after shRNA transfection with or without LPS treatment. F The co-IP assay was carried out in HepG2-shCTR and HepG2 cells after shRNA transfection with or without LPS treatment for verifying the interaction capacity of Caspase-8 to Myd88, nmMYLK, and IRAK4. Data were shown as mean \pm SD from three independent experiments. ${ }^{* *} P<0.01$.

\section{Flow cytometric analysis}

The early apoptosis rates in 7703 and HepG2 cells under the indicated treatments were stained with Annexin-V/7-AAD according to manufacturer's instructions and then subjected to flow cytometry.

\section{Tumorigenicity experiments}

Cells were suspended in Matrigel/PBS (1:1) at a concentration of $5 \times 10^{7}$ cells $/ \mathrm{ml}$. The cell mixture was injected subcutaneously into the left/right back of the mice $(0.1 \mathrm{ml} /$ per mouse). After 4 weeks, all mice were sacrificed, and the tumors were dissected. Tumor size was measured and calculated using the equation (length $\times$ width $\left.^{2}\right) / 2$.

\section{Immunofluorescence}

Cells were cultured for $24 \mathrm{~h}$ on culture slides and washed in PBS after adhering to slides. Then, the fixed cells were treated with $10 \%$ Goat serum albumin and incubated with primary antibody overnight. Following washing, secondary antibody was added, the slides were stained in DAPI (Vector Laboratories, CA, USA) and examined by confocal microscope.

\section{Western blot analysis}

Tumor cells were lysed in the lysis buffer with a protease and phosphatase inhibitor cocktail. Western blotting was performed as standard protocol. Immobilon Western Chemiluminescent HRP Substrate (WBKLS0500, Millipore) was used for visualization and the signal was analyzed with Alpha Ease FC software (Bio-Rad, Hercules, CA).

\section{Co-immunoprecipitation (Co-IP)}

Cells were treated with IP lysis buffer, then lysates were probed with antibody and magnetic beads at $4^{\circ} \mathrm{C}$ all night. After that, samples were washed in PBS and eluted for western blot.

\section{Immunohistochemistry (IHC) analysis and TUNEL staining}

Immunohistochemical analysis was performed as described previously [47]. In brief, tumor tissues were fixed in $4 \%$ paraformaldehyde and embedded in paraffin. Sections $(4 \mu \mathrm{m})$ were prepared for indicated primary antibodies or hematoxylin and eosin (H\&E) staining according to standard protocols. TUNEL Apoptosis Assay Kit was applied to validate the apoptotic cells according to manufacturers' instructions (C1091, Beyotime, China). 


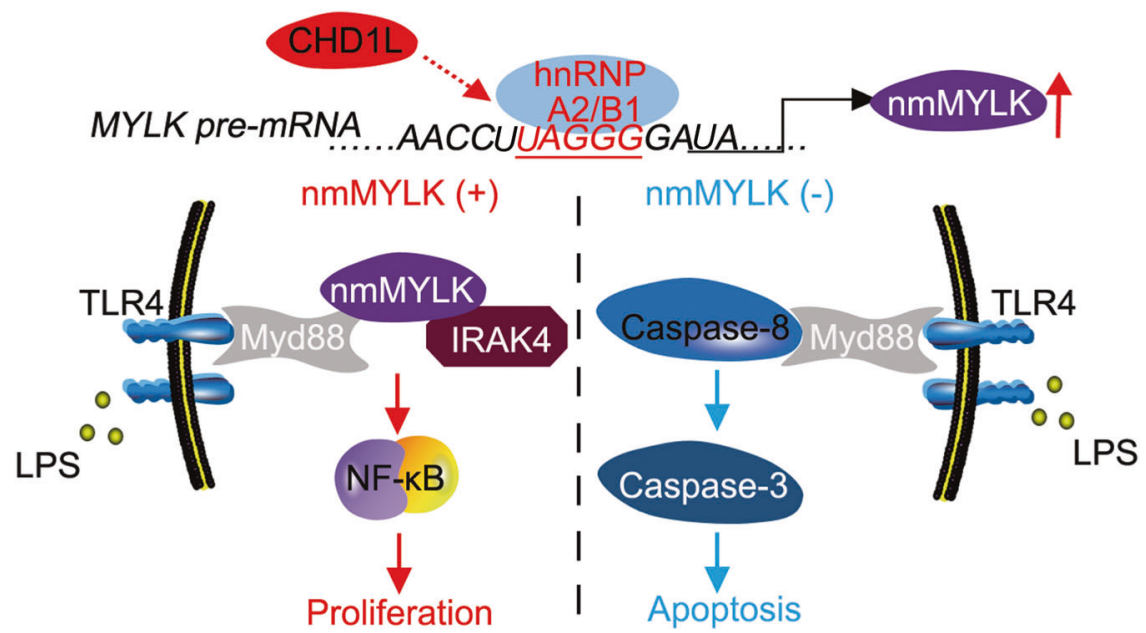

Fig. 7 Schematic diagram depicts a proposed model for the regulation and mechanism of nmMYLK in HCC. CHD1L upregulates hnRNP A2/ $\mathrm{B} 1$ to promise the nmMYLK formation. Abundant nmMYLK might interact with MyD88 and IRAK4 to activate NF- $\mathrm{nB}$ and its downstream signaling, which finally promoting the proliferation of tumor cells. The Depleted nmMYLK enable MyD88 to interact with Caspase-8 and lead to the cascade activation reaction of caspase proteins, which finally result in tumor cell apoptosis.

\section{Cytosolic $\mathrm{Ca}^{2+}$ measurements}

The intracellular $\mathrm{Ca}^{2+}$ concentration was calculated using Fura-4 $\mathrm{Ca}^{2+}$ Imaging Calibration kit (Invitrogen) according to the manufacturer's protocol and equation.

\section{RT-PCR and quantitative real-time PCR (qRT-PCR)}

Total RNA was extracted using TRIZOL Reagent (\#15596-018, Invitrogen) and was reversed transcribed using CDNA reverse transcription kit (\#RR037A, Takara). According to the manufacturer's instructions, qRT-PCR with SYBR Green Master Mix (Vazyme) on StepOnePlus real-time PCR system (ABI). The primers used were shown in Table S2.

\section{RNA immunoprecipitation (RIP) assay}

After culturing in RIP lysis buffer, lysates from processed cells were conjugated with antibody or control lgG antibody in magnetic beads. After RNA enrichment, qRT-PCR was implemented to analyze RNA enrichment. The primers used were listed in Table S3.

\section{Statistical analysis}

All data were presented as mean \pm SD. Student's $t$-test (two groups) or oneway ANOVA (multiple groups) were applied to analyze the differences between groups by using SPSS 22.0. $P$ values less than 0.05 were considered statistically significant.

\section{DATA AVAILABILITY}

The datasets used and/or analyzed during the current study are available from the corresponding author on reasonable request.

\section{REFERENCES}

1. Ma NF, Hu L, Fung JM, Xie D, Zheng BJ, Chen L, et al. Isolation and characterization of a novel oncogene, amplified in liver cancer 1 , within a commonly amplified region at 1q21 in hepatocellular carcinoma. Hepatology. 2008;47:503-10.

2. Liu M, Chen $L, M a N F$, Chow RK, Li $Y$, Song $Y$, et al. CHD1L promotes lineage reversion of hepatocellular carcinoma through opening chromatin for key developmental transcription factors. Hepatology. 2016;63:1544-59.

3. He WP, Guo YY, Yang GP, Lai HL, Sun TT, Zhang ZW, et al. CHD1L promotes EOC cell invasiveness and metastasis via the regulation of METAP2. Int J Med Sci. 2020;17:2387-95.

4. Tian F, Xu F, Zhang ZY, Ge JP, Wei ZF, Xu XF, et al. Expression of CHD1L in bladder cancer and its influence on prognosis and survival. Tumour Biol. 2013;34:3687-90.

5. Ji X, Li J, Zhu L, Cai J, Zhang J, Qu Y, et al. CHD1L promotes tumor progression and predicts survival in colorectal carcinoma. J Surg Res. 2013;185:84-91.

6. Wang W, Wu J, Fei X, Chen W, Li Y, Shen K, et al. CHD1L promotes cell cycle progression and cell motility by up-regulating MDM2 in breast cancer. Am J Transl Res. 2019;11:1581-92.
7. He LR, Ma NF, Chen JW, Li BK, Guan XY, Liu MZ, et al. Overexpression of CHD1L is positively associated with metastasis of lung adenocarcinoma and predicts patients poor survival. Oncotarget. 2015;6:31181-90.

8. Ning $X$, Deng $Y$. Identification of key pathways and genes influencing prognosis in bladder urothelial carcinoma. Onco Targets Ther. 2017;10:1673-86.

9. Chen L, Su L, Li J, Zheng Y, Yu B, Yu Y, et al. Hypermethylated FAM5C and MYLK in serum as diagnosis and pre-warning markers for gastric cancer. Dis Markers. 2012;32:195-202.

10. Minamiya $Y$, Nakagawa $T$, Saito $H$, Matsuzaki I, Taguchi $K$, Ito $M$, et al. Increased expression of myosin light chain kinase mRNA is related to metastasis in nonsmall cell lung cancer. Tumour Biol. 2005;26:153-7.

11. Kamm KE, Stull JT. Dedicated myosin light chain kinases with diverse cellular functions. J Biol Chem. 2001;276:4527-30.

12. Tauseef M, Knezevic N, Chava KR, Smith M, Sukriti S, Gianaris N, et al. TLR4 activation of TRPC6-dependent calcium signaling mediates endotoxin-induced lung vascular permeability and inflammation. J Exp Med. 2012;209:1953-68.

13. Dreyfuss G, Matunis MJ, Piñol-Roma S, Burd CG. hnRNP proteins and the biogenesis of mRNA. Annu Rev Biochem. 1993;62:289-321.

14. Kozu T, Henrich B, Schäfer KP. Structure and expression of the gene (HNRPA2B1) encoding the human hnRNP protein A2/B1. Genomics. 1995;25:365-71.

15. Hu Y, Sun Z, Deng J, Hu B, Yan W, Wei H, et al. Splicing factor hnRNPA2B1 contributes to tumorigenic potential of breast cancer cells through STAT3 and ERK1/2 signaling pathway. Tumour Biol. 2017;39:1010428317694318.

16. Dai L, Li J, Tsay JJ, Yie TA, Munger JS, Pass H, et al. Identification of autoantibodies to ECH1 and HNRNPA2B1 as potential biomarkers in the early detection of lung cancer. Oncoimmunology. 2017;6:e1310359.

17. Mascarenhas JB, Tchourbanov AY, Danilov SM, Zhou T, Wang T, Garcia J. The splicing factor hnRNPA1 regulates alternate splicing of the MYLK gene. Am J Respir Cell Mol Biol. 2018;58:604-13.

18. Huelga SC, Vu AQ, Arnold JD, Liang TY, Liu PP, Yan BY, et al. Integrative genomewide analysis reveals cooperative regulation of alternative splicing by hnRNP proteins. Cell Rep. 2012;1:167-78.

19. Wu B, Su S, Patil DP, Liu H, Gan J, Jaffrey SR, et al. Molecular basis for the specific and multivariant recognitions of RNA substrates by human hnRNP A2/B1. Nat Commun. 2018;9:420.

20. Wang T, Moreno-Vinasco L, Ma SF, Zhou T, Shimizu Y, Sammani S, et al. Nonmuscle myosin light chain kinase regulates murine asthmatic inflammation. Am J Respir Cell Mol Biol. 2014;50:1129-35.

21. Mirzapoiazova T, Moitra J, Moreno-Vinasco L, Sammani S, Turner JR, Chiang ET, et al. Non-muscle myosin light chain kinase isoform is a viable molecular target in acute inflammatory lung injury. Am J Respir Cell Mol Biol. 2011:44:40-52.

22. Dai W, Wu J, Zhang S, Shi B, Xu X, Wang D, et al. Genes directly regulated by NF-KB in human hepatocellular carcinoma HepG2. Int J Biochem Cell Biol. 2017:89:157-70.

23. Eisen JA, Sweder KS, Hanawalt PC. Evolution of the SNF2 family of proteins: subfamilies with distinct sequences and functions. Nucleic Acids Res. 1995;23:2715-23.

24. Singh HR, Nardozza AP, Möller IR, Knobloch G, Kistemaker $H$, Hassler $M$, et al. A poly-ADP-ribose trigger releases the auto-inhibition of a chromatin remodeling oncogene. Mol Cell. 2017;68:860-71. 
25. Chen L, Chan TH, Yuan YF, Hu L, Huang J, Ma S, et al. CHD1L promotes hepatocellular carcinoma progression and metastasis in mice and is associated with these processes in human patients. J Clin Invest. 2010;120:1178-91.

26. Chan TH, Chen L, Liu M, Hu L, Zheng BJ, Poon VK, et al. Translationally controlled tumor protein induces mitotic defects and chromosome missegregation in hepatocellular carcinoma development. Hepatology. 2012;55:491-505.

27. Li Y, Chen L, Chan TH, Liu M, Kong KL, Qiu JL, et al. SPOCK1 is regulated by CHD1L and blocks apoptosis and promotes HCC cell invasiveness and metastasis in mice. Gastroenterology. 2013;144:179-91.

28. Chen L, Hu L, Chan TH, Tsao GS, Xie D, Huo KK, et al. Chromodomain helicase/ adenosine triphosphatase DNA binding protein 1-like (CHD1l) gene suppresses the nucleus-to-mitochondria translocation of nur77 to sustain hepatocellular carcinoma cell survival. Hepatology. 2009;50:122-9.

29. Xia N, Cui J, Zhu M, Xing R, Lu Y. Androgen receptor variant 12 promotes migration and invasion by regulating MYLK in gastric cancer. J Pathol. 2019;248:304-15.

30. Han YJ, Ma SF, Yourek G, Park YD, Garcia JG. A transcribed pseudogene of MYLK promotes cell proliferation. FASEB J. 2011;25:2305-12.

31. Lin J, He Y, Chen L, Chen X, Zang S, Lin W. MYLK promotes hepatocellular carcinoma progression through regulating cytoskeleton to enhance epithelialmesenchymal transition. Clin Exp Med. 2018;18:523-33.

32. Srivastava N, Tauseef M, Amin R, Joshi B, Joshi JC, Kini V, et al. Noncanonical function of long myosin light chain kinase in increasing ER-PM junctions and augmentation of SOCE. FASEB J. 2020;34:12805-19.

33. Keller N, Ozmadenci D, Ichim G, Stupack D. Caspase-8 function, and phosphorylation, in cell migration. Semin Cell Dev Biol. 2018;82:105-17.

34. Glisovic T, Bachorik JL, Yong J, Dreyfuss G. RNA-binding proteins and posttranscriptional gene regulation. FEBS Lett. 2008;582:1977-86.

35. Keene JD. RNA regulons: coordination of post-transcriptional events. Nat Rev Genet. 2007;8:533-43.

36. Lei Y, Guo W, Chen B, Chen L, Gong J, Li W. Tumor-released IncRNA H19 promotes gefitinib resistance via packaging into exosomes in non-small cell lung cancer. Oncol Rep. 2018;40:3438-46.

37. Yang J, Chen Y, Lu J, Wang X, Wang L, Liang J, et al. Identification and characterization of novel fusion genes in prostate cancer by targeted RNA capture and nextgeneration sequencing. Acta Biochim Biophys Sin (Shanghai). 2018;50:1166-72.

38. Dai S, Zhang J, Huang S, Lou B, Fang B, Ye T, et al. HNRNPA2B1 regulates the epithelial-mesenchymal transition in pancreatic cancer cells through the ERK snail signalling pathway. Cancer Cell Int. 2017;17:12.

39. Wang H, Liang L, Dong Q, Huan L, He J, Li B, et al. Long noncoding RNA miR503HG, a prognostic indicator, inhibits tumor metastasis by regulating the HNRNPA2B1/NFKB pathway in hepatocellular carcinoma. Theranostics. 2018;8:2814-29.

40. Takeuchi O, Akira S. Pattern recognition receptors and inflammation. Cell. 2010;140:805-20.

41. Mehta $D$, Malik $A B$. Signaling mechanisms regulating endothelial permeability. Physiol Rev. 2006;86:279-367.

42. Lin SC, Lo YC, Wu H. Helical assembly in the MyD88-IRAK4-IRAK2 complex in TLR/ IL-1R signalling. Nature. 2010;465:885-90.

43. Zhou H, Harberts E, Fishelevich R, Gaspari AA. TLR4 acts as a death receptor for ultraviolet radiation (UVR) through IRAK-independent and FADD-dependent pathway in macrophages. Exp Dermatol. 2016;25:949-55.

44. Graf R, Barbero S, Keller N, Chen L, Uryu S, Schlaepfer D, et al. Src-inducible association of CrkL with procaspase-8 promotes cell migration. Cell Adh Migr. 2013;7:362-9.

45. Lin P, Bush JA, Cheung KJ, Li G. Tissue-specific regulation of Fas/APO-1/CD95 expression by p53. Int J Oncol. 2002;21:261-4.

46. Gdynia G, Grund K, Eckert A, Böck BC, Funke B, Macher-Goeppinger S, et al. Basal caspase activity promotes migration and invasiveness in glioblastoma cells. Mol Cancer Res. 2007:5:1232-40.

47. Peng $H$, Liu J, Sun $Q$, Chen R, Wang $Y$, Duan J, et al. mTORC1 enhancement of STIM1-mediated store-operated $\mathrm{Ca} 2+$ entry constrains tuberous sclerosis complex-related tumor development. Oncogene. 2013;32:4702-11.

\section{ACKNOWLEDGEMENTS}

We appreciate the supports of our experimenters, we thanks to Professor Guan-Xing Yuan for the material supporting.

\section{AUTHOR CONTRIBUTIONS}

GW, XZ, WC, YM, JC, ZC, XC, and HC performed the experiments; GW, XZ, SL, and LH analyzed the data and performed statistical analyses; ML and LM provided technical, material support, and valuable comments; GW, XZ, and NFM wrote the manuscript; NFM designed and supervised the study and made critical corrections; and all authors reviewed and approved the manuscript.

\section{FUNDING}

This work was funded by the Natural Science Foundation of Guangdong Province (2017A030313562), the Science and Technology Program of Guangzhou (2018040100160), Guangzhou key medical discipline construction project fund.

\section{ETHICS APPROVAL}

Our research was approved by the Ethics Committee of Guangzhou Medical University and all experiments were performed in accordance with relevant guidelines and regulations.

\section{COMPETING INTERESTS}

The authors declare no competing interests.

\section{ADDITIONAL INFORMATION}

Supplementary information The online version contains supplementary material available at https://doi.org/10.1038/s41419-021-04167-9.

Correspondence and requests for materials should be addressed to Ning-Fang Ma.

Reprints and permission information is available at http://www.nature.com/ reprints

Publisher's note Springer Nature remains neutral with regard to jurisdictional claims in published maps and institutional affiliations.

\begin{abstract}
Open Access This article is licensed under a Creative Commons Attribution 4.0 International License, which permits use, sharing, adaptation, distribution and reproduction in any medium or format, as long as you give appropriate credit to the original author(s) and the source, provide a link to the Creative Commons license, and indicate if changes were made. The images or other third party material in this article are included in the article's Creative Commons license, unless indicated otherwise in a credit line to the material. If material is not included in the article's Creative Commons license and your intended use is not permitted by statutory regulation or exceeds the permitted use, you will need to obtain permission directly from the copyright holder. To view a copy of this license, visit http://creativecommons. org/licenses/by/4.0/.
\end{abstract}

(c) The Author(s) 2021, corrected publication 2022 